\title{
SEVERE CMD WITH NOVEL MUTATION IN LAMIN A/C GENE
}

Researchers at Queen Fabiola Children's University Hospital, Brussels, Belgium, report a 7-year-old Belgian boy with a 5 months-onset congenital muscular dystrophy and laminopathy caused by a de novo heterozygous LMNA gene mutation. Muscle weakness and wasting affected the axial muscles, proximal upper and distal lower extremities, with rapid development of joint contractures and spine rigidity. Serum CK was moderately elevated. Muscle biopsy showed a dystrophic pattern with normal immunochemical studies, excluding classic forms of CMD. (Prigogine C, Richard P, Van den Bergh P, Groswasser J, Deconinck N. Novel LMNA mutation presenting as severe congenital muscular dystrophy. Pediatr Neurol October 2010;43:283-286). (Respond: Dr Deconinck, Department of Neurology, Universite Libre de Bruxelles, 1020 Brussels, Belgium. E-mail: Nicolas.deconinck@huderf.be).

COMMENT. Previous reports of 15 cases of CMD with LMNA mutations (Quijano-Roy et al. Ann Neurol 2008;64:177-186) cited by the authors distinguish two groups, I $(n=30)$ a severe group with absent motor development, and II $(n=12)$, a dropped-head syndrome phenotype with a milder disease in children who initially sat and walked, and then developed neck weakness. Laminopathies are a heterogeneous group of diseases that include autosomal dominant Emery-Dreifuss muscular dystrophy and limbgirdle muscular dystrophy type 1B.

\section{ATYPICAL CHRONIC INFLAMMATORY DEMYELINATING POLYNEUROPATHY}

Researchers at the Royal Children's Hospital and Melbourne University, Victoria, Australia report a 12-year-old boy with a 12-month history of progressive weakness affecting extremities and neck flexion but no facial weakness. Gowers' sign was positive. Reflexes were diminished or absent. Sensation was normal. Nerve conduction was nomal. EMG revealed decreased recruitment and high-amplitude poyphasic motor unit potentials. Biopsy of vastus lateralis showed grouped fiber atrophy and target fiber formation. CSF showed minimally elevated protein of $0.51 \mathrm{~g} / \mathrm{L}$. MRI spine showed no enlargement of norve roots. Repeat neurophysiologica! testing 5 months later and with further progression of weakness showed an increase in peroneal distal latencies and slowing of tibial motor nerve conduction velocity. The findings were insufficient to meet criteria for chronic inflammatory demyelinating polyneuropathy (CIDP) but an acquired demyelinating polyneuropathy was suspected, and treatment with IVIg was begun. Improvement was noted within 1 week, and at 1-year follow-up, 6 months after the last infusion, he was $80 \%$ recovered. Despite absence of all typical signs of CIDP, an empiric trial of immunomodulatory therapy was both diagnostically and therapeutically valuable. (Mohamad AR, Rodriguez-Casero MV, Ryan MM. Atypical childhood chronic inflammatory demyelinating polyneuropathy. Muscle Nerve August 2010;42:293295).(Respond: Dr Monique M Ryan. E-mail: monique.ryan@rch.org.au).

COMMENT. A trial of IVIg may be indicated in clinically suspected CIDP cases that lack other diagnostic criteria, including NCV, EMG, CSF, MRI, and nerve biopsy. 